\title{
Bioinformatics analysis to identify key genes and pathways influencing synovial inflammation in osteoarthritis
}

\author{
JIE LIN $^{1 *}$, GUANGWEN WU ${ }^{2 *}$, ZHONGSHENG ZHAO $^{1}$, YANFENG HUANG ${ }^{1}$, \\ JUN CHEN $^{3}$, CHANGLONG FU ${ }^{1}$, JINXIA YE ${ }^{2}$ and XIANXIANG LIU ${ }^{1}$ \\ ${ }^{1}$ Academy of Integrative Medicine, Fujian University of Traditional Chinese Medicine; \\ ${ }^{2}$ Fujian Provincial Key Laboratory of Integrative Medicine on Geriatrics; ${ }^{3}$ College of Integrative Medicine, \\ Fujian University of Traditional Chinese Medicine, Fuzhou, Fujian 350122, P.R. China
}

Received April 24, 2018; Accepted September 14, 2018

DOI: $10.3892 / \mathrm{mmr} .2018 .9575$

\begin{abstract}
Osteoarthritis (OA) is a chronic arthropathy that occurs in the middle-aged and elderly population. The present study aimed to identify gene signature differences between synovial cells from OA synovial membrane with and without inflammation, and to explain the potential mechanisms involved. The differentially expressed genes (DEGs) between 12 synovial membrane with inflammation and 12 synovial membrane without inflammation from the dataset GSE46750 were identified using the Gene Expression Omnibus 2R. The DEGs were subjected to enrichment analysis, protein-protein interaction (PPI) analysis and module analysis. The analysis results were compared with text-mining results. A total of 174 DEGs were identified. Gene Ontology enrichment results demonstrated that functional molecules encoded by the DEGs primarily had extracellular location, molecular functions predominantly involving 'chemokine activity' and 'cytokine activity', and were associated with biological processes, including 'inflammatory response' and 'immune response'. The Kyoto Encyclopedia of Genes and Genomes results demonstrated that DEGS may function through pathways associated with 'rheumatoid arthritis', 'chemokine signaling pathway', 'complement and coagulation cascades', 'TNF signaling pathway', 'intestinal immune networks for IgA production', 'cytokine-cytokine receptor interaction', 'allograft rejection', 'Toll-like receptor signaling pathway' and 'antigen processing and presentation'. The top 10 hub genes [interleukin (IL)6, IL8, matrix metallopeptidase (MMP)9, colony stimulating factor 1 receptor, FOS proto-oncogene,
\end{abstract}

Correspondence to: Professor Xianxiang Liu, Academy of Integrative Medicine, Fujian University of Traditional Chinese Medicine, 1 Qiuyang Road, Fuzhou, Fujian 350122, P.R. China E-mail: liuxianxiang@163.com

*Contributed equally

Key words: bioinformatics analysis, osteoarthritis, inflammatory, microarray, differentially expressed gene
AP1 transcription factor subunit, insulin-like growth factor 1, TYRO protein tyrosine kinase binding protein, MMP3, cluster of differentiation (CD)14 and CD163] and four gene modules were identified from the PPI network using Cytoscape. In addition, text-mining was used to identify the commonly used drugs and their targets for the treatment of OA. It was initially verified whether the results of the present study were useful for the study of OA treatment targets and pathways. The present study provided insight for the molecular mechanisms of OA synovitis. The hub genes and associated pathways derived from analysis may be targets for OA treatment. IL8 and MMP9, which were validated by text-mining, may be used as molecular targets for the OA treatment, while other hub genes require further validation.

\section{Introduction}

Osteoarthritis (OA) is one of the most common types of chronic arthritis in elderly people, and has been identified to affect $\geq 6-32 \%$ people worldwide (1). There are multiple risk factors for OA development, including abnormal mechanical stress, aging, obesity and genetic factors (2). Pathological features of OA primarily include articular cartilage degeneration, synovial inflammation and atypical bone formation (3). In the past, research has primarily focused on cartilage tissue and chondrocytes in the study of OA mechanisms and treatment $(4,5)$. With the rapid development of medicine, synovial tissue and subchondral bone have additionally been investigated. Previous studies suggested that synovial tissue serves an important role in OA $(6,7)$. As part of the joint structure, the synovial membrane may produce and regulate synovial fluid, maintain joint activity, and be adversely affected in joint diseases (8). Synovial lesions may additionally be identified in a number of joint diseases, which may serve a role in promoting the occurrence and progression of the disease (9).

Synovitis is an aseptic inflammation that occurs at the synovial membrane and is observed in rheumatoid arthritis, OA, lupus OA and gout (10). Synovitis leads to abnormal synovial fluid production and absorption, resulting in joint effusion, causing pain, swelling and other reactions (11). Previous studies observed synovitis in early OA (12), and that the severity of synovitis may be associated with different 
stages of OA (13). Fernandez-Madrid et al (14) suggested that OA synovitis is caused by the degeneration of cartilage stimulation. However, Felson et al (15) suggested that synovitis occurs not only in the early stages of OA; however, even prior to imaging. Additionally, the occurrence of synovitis may further promote cartilage degeneration, which would in turn exacerbate synovitis (11). Synovitis serves an important role in the symptoms, progression and development of OA, and is a concern for the treatment of OA.

With the development of modern biomedicine, increasing evidence suggested that the occurrence and development of OA may be mediated by a number of genes and signaling pathways (16). In order to develop clearer diagnostic criteria and more effective treatment options, it is essential to fully understand the molecular mechanism of OA. With the aim of fully understanding the gene expression alterations in OA, previous studies used DNA microarray technology to analyze gene expression profiles $(17,18)$. The results demonstrated that molecules encoded by differentially expressed genes (DEGs) located in different cell structures and with different molecular functions (MF) were associated with different biological processes (BP) during their involvement in the disease process. The availability of bioinformatics analysis based on high-throughput technology enabled the investigation of the alterations in mRNA expression and the interaction between differential genes in $\mathrm{OA}$, to provide novel insights for further in-depth OA studies.

The Gene Expression Omnibus (GEO) is a database and online resource for the gene expression of any species. The present study obtained genetic microarray dataset no. GSE46750 from GEO. The samples in GSE46750 were divided into two groups: Synovial cells with and without inflammation in OA. The two groups were compared and analyzed to identify the DEGs. Functional enrichment analysis, protein-protein interaction (PPI) networks and module analysis were conducted on the DEGs. Subsequently, text-mining of OA treatment drugs and their target genes were performed to initially validate the results. The results of the present study may enable us to recognize the effects of synovial membrane inflammation in the development of OA, and to provide certain possible OA target molecules for subsequent validation.

\section{Materials and methods}

Gene chip data. GSE46750 gene expression data (19) was obtained from the GEO database (http://www.ncbi.nlm.nih. gov/geo/), which was expressed on the GPL10558 platform [(Illumina HumanHT-12 V 4.0) Bead chip; Illumina, Inc., San Diego, CA, USA]. The GSE46750 dataset samples, which were synovial cells, were derived from 12 patients with OA, specifically from those with synovial membrane with inflammation $(n=12)$ and synovial membrane without inflammation $(n=12)$.

Identifying DEGs. The original micro array data was analyzed through heat mapping using Morpheus (https://software. broadinstitute.org/morpheus/) to visually observe gene expression. The chip data were divided into an inflammatory synovial membrane group and a non-inflammatory synovial membrane group for analysis. GEO2R (https://www.ncbi.nlm. nih.gov/geo/geo2r/?acc=GSE46750) was used to identify the
DEGs in OA synovial membrane. The criteria for a DEG was $\mid \log 2$ (fold change) $1 \geq 1$ and $\mathrm{P}<0.05$.

Gene Ontology (GO) enrichment and Kyoto Encyclopedia of Genes and Genomes (KEGG) pathway analysis. GO enrichment analysis enables the annotation of cell components (CC), $\mathrm{MF}$ and BP of DEGs. KEGG (http://www.genome.jp/) is a database that includes a KEGG path database for examining the path of the gene cluster and associated functions. The present study used Database for Annotation, Visualization and Integrated Discovery (DAVID 6.8; https://david.ncifcrf. gov/) (20) to perform GO enrichment analysis and KEGG pathway analysis. $\mathrm{P}<0.05$ was considered to indicate a statistically significant difference.

Construction of the PPI network and module analysis. The Search Tool for the Retrieval of Interacting Genes/Proteins (STRING) database (http://www.string-db.org/) may be searched for associations between known and predicted proteins, and is commonly used to predict PPI information in molecular biology (21). The DEGs were mapped to STRING, and those with interaction scores $>0.4$ were selected for further examination. Cytoscape 3.5.0 (http://www.cytoscape.org/) was used to visualize the results from the PPI network. Module analysis on the PPI network results was performed using the molecular complex detection (MCODE) (22) clustering algorithm that comes with Cytoscape. Module analysis may be used to identify more connected gene groups. In addition, the module analysis results were further analyzed for function and pathway enrichment, and $\mathrm{P}<0.05$ was considered to indicate a statistically significant difference.

Text-mining. Drugs commonly used for the treatment of OA were searched in The Therapeutic Target Database (https://db.idrblab.org/ttd/) (23) with the key word 'osteoarthritis'. The molecular targets of the detected drugs were identified in The Drug Gene Interaction database (DGIdb; http://dgidb.genome.wustl.edu/). These known molecules that may serve a therapeutic role in OA were compared with the present results.

\section{Results}

DEGs. Morpheus was used to observe the overall gene expression of all samples (Fig. 1), and differences in gene expression between inflammatory synovial cells $(n=12)$ and non-inflammatory synovial cells $(n=12)$ were identified. A total of 174 DEGs were identified by GEO2R analysis, of which 145 were upregulated and 29 were downregulated (Fig. 2).

GO term enrichment analysis. Fig. 3 presents the results of the GO analysis. The majority of the DEGs were determined to be located in the 'extracellular region', the 'plasma membrane' and the 'vesicle'. The DEGs were demonstrated to exert molecular functions by regulating 'chemokine activity', 'chemokine receptor binding', 'growth factor activity' and other 'cytokine activity'. Furthermore, the DEGs may regulate the OA membrane inflammatory response by involvement in the regulation of 'inflammatory response', 'immune response', 'response to lipopolysaccharide' and 'chemotaxis'. 


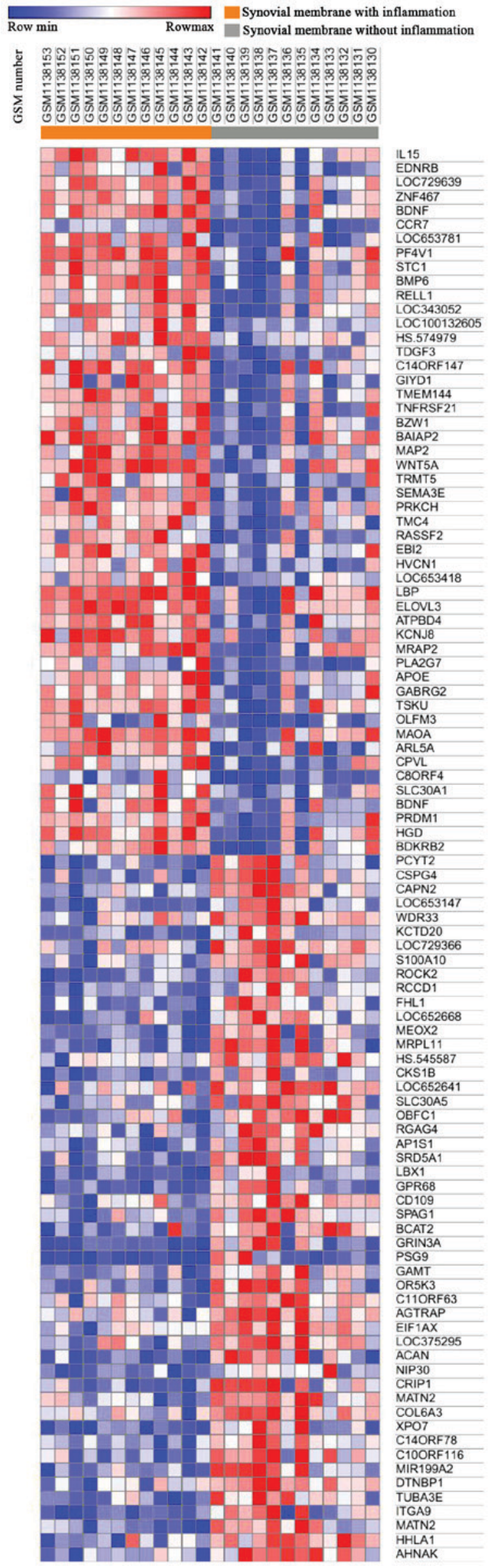

Figure 1. Heat map of 100 genes from all samples. Red indicates higher gene expression and blue indicates lower gene expression.

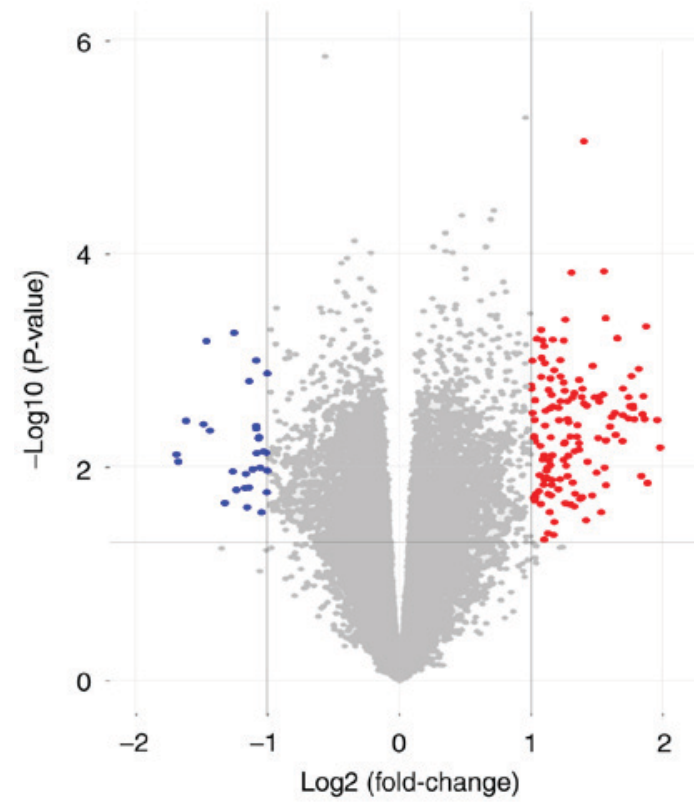

Figure 2. Volcanic map of all genes. Red dots indicate upregulated genes, blue dots indicate downregulated genes and gray dots indicate genes that are not regulated.

KEGG pathway analysis. The KEGG analysis results (Fig. 4) suggested that DEGs, through pathways, including 'chemokine signaling pathway', 'complement and coagulation cascades', 'TNF signaling pathway', 'intestinal immune network for IgA production', 'cytokine-cytokine receptor interaction', 'Toll-like receptor signaling pathway' and 'antigen processing and presentation', serve a role in regulating the synovial inflammation of $\mathrm{OA}$.

Analysis of hub genes and modules. Following the analysis based on the PPI networks, 122 nodes (DEGs) and 444 edges (interactions between DEGs) were identified in Cytoscape (Fig. 5). The genes with higher scores were the hub genes, as the genes of higher degree may be associated with OA. The top 10 hub genes were interleukin (IL)6, IL8, matrix metallopeptidase (MMP)9, colony stimulating factor 1 receptor (CSF1R), FOS proto-oncogene, AP1 transcription factor subunit (FOS), insulin-like growth factor 1 (IGF1), TYRO protein tyrosine kinase binding protein (TYROBP), MMP3, cluster of differentiation (CD) 14 and CD163. A total of four modules were selected through MCODE analysis (Fig. 5). Enrichment analysis demonstrated that modules 1 and 2 may be associated with 'cytokine-cytokine receptor interactions', 'NOD-like receptor signaling pathways' and 'toll-like receptor signaling pathways' (Table I).

Common therapeutic drugs and their targets. The drugs commonly used in the treatment of OA included lumiracoxib, rofecoxib, guggulsterone, nepafenac, glucosamine, diclofenac, valdecoxib, naproxen, tiaprofenic acid, celecoxib, tolmetin, etoricoxib, tenoxicam, salsalate and mefenamic acid (Fig. 6). The target genes of these drugs were identified in DGIdb, which included IL8, MMP9, IL10, BCL2 associated $\mathrm{X}$, apoptosis regulator, cyclin dependent kinase inhibitor 1B (Fig. 6). 


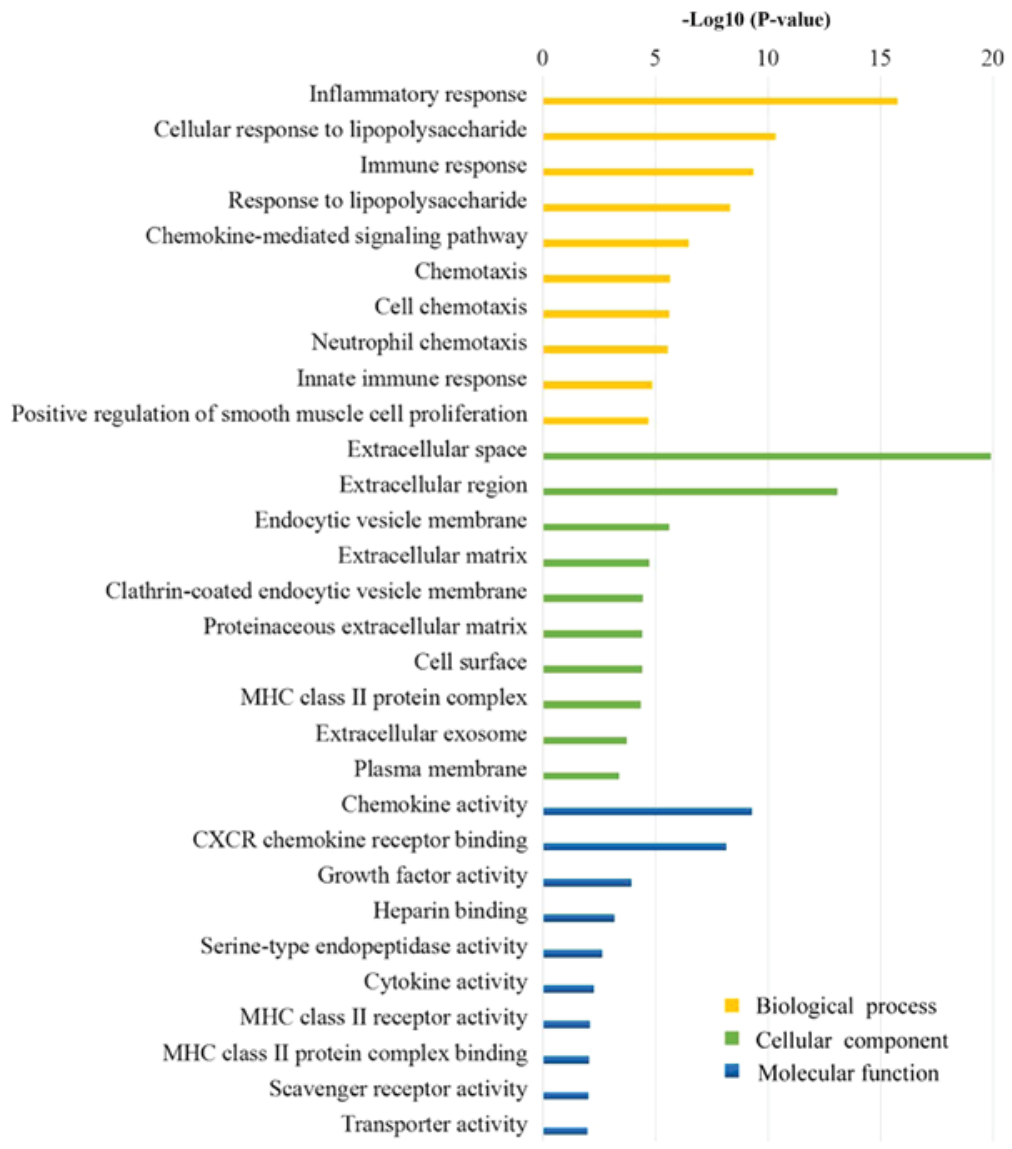

Figure 3. The top 10 biological processes, cellular component and molecular function analysis in differentially expressed genes between non-inflammatory synovial cells and inflammatory synovial cells in osteoarthritis.

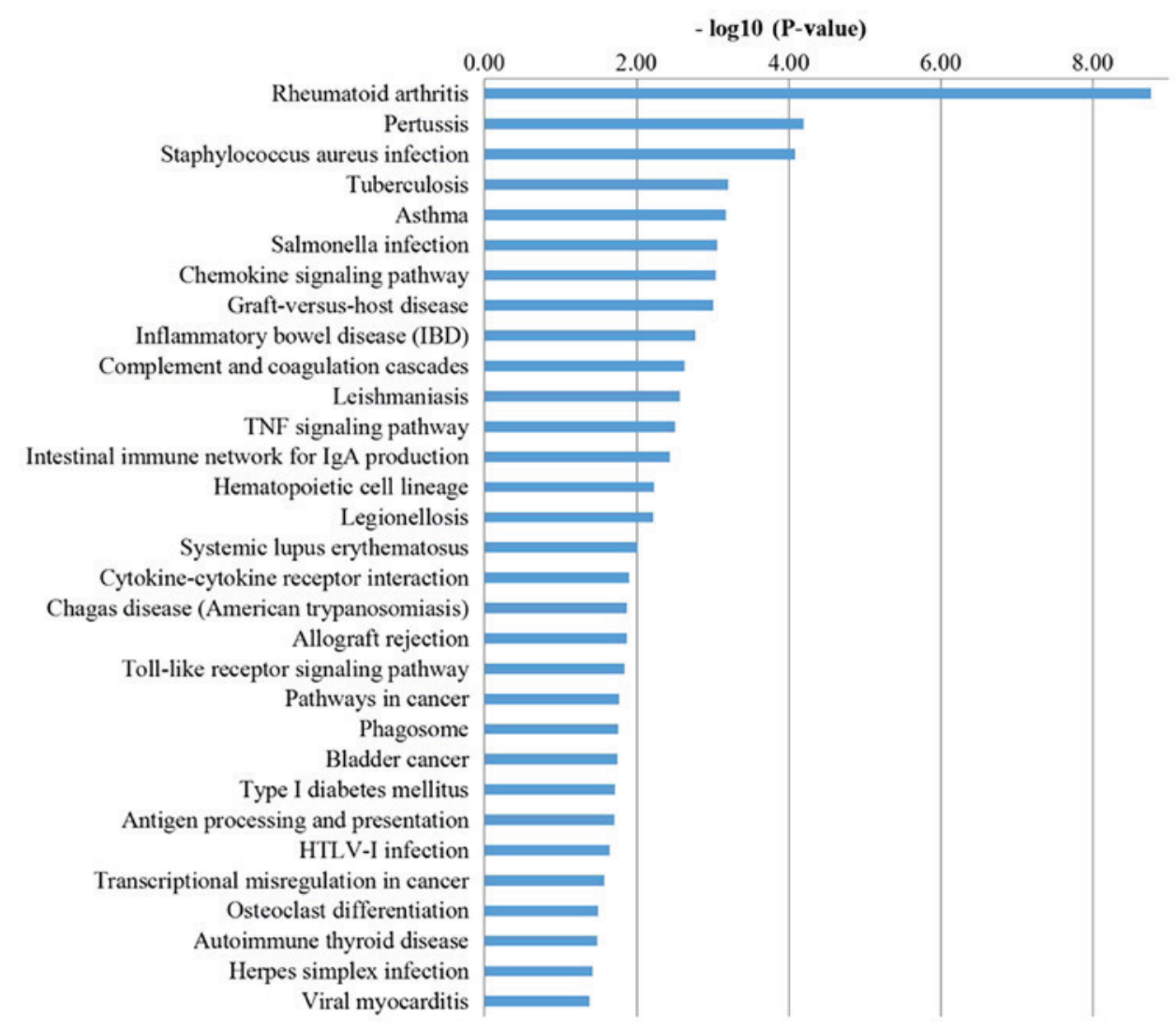

Figure 4. Kyoto Encyclopedia of Genes and Genomes pathways in differentially expressed genes between non-inflammatory synovial cells and inflammatory synovial cells in osteoarthritis. 


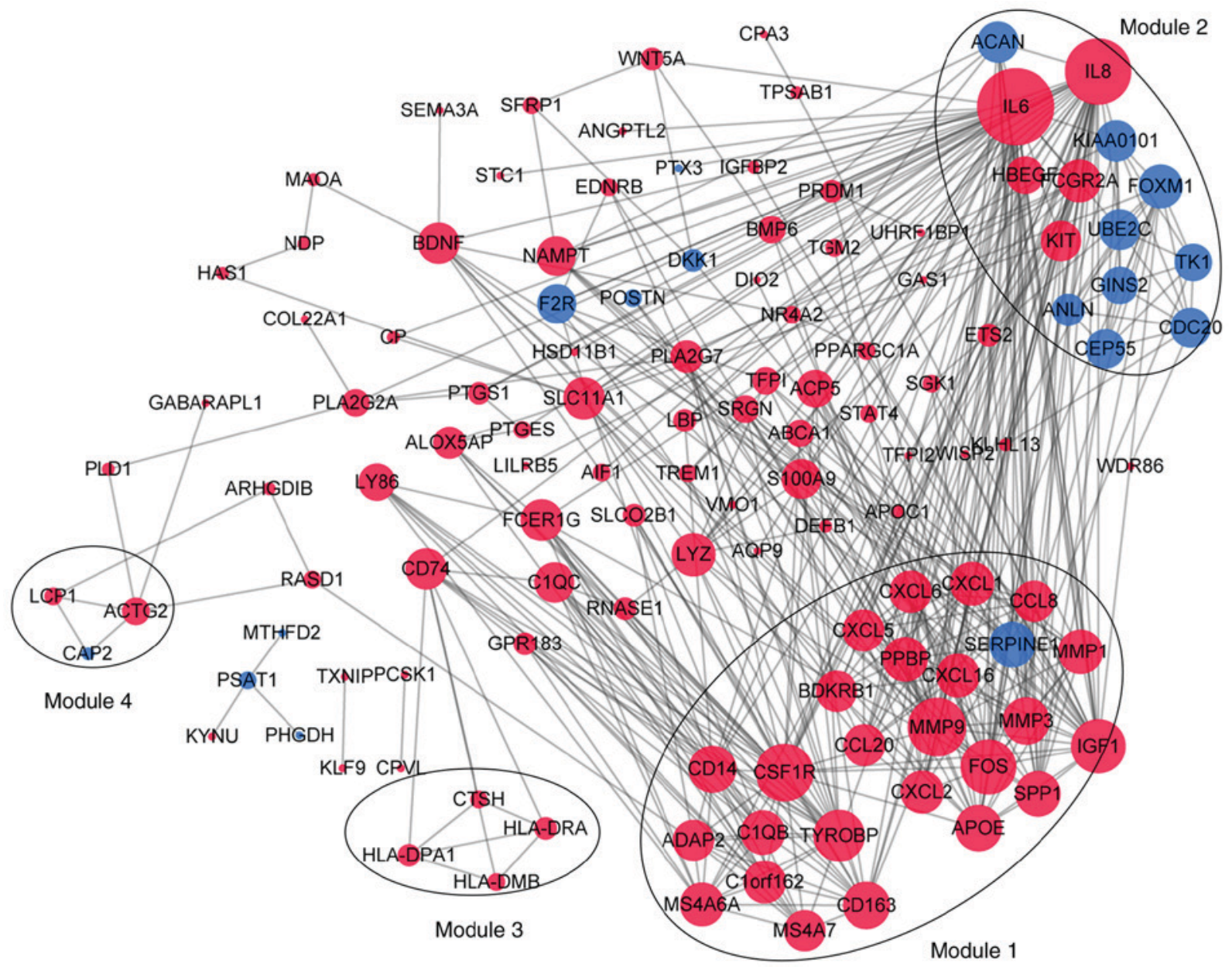

Figure 5. Protein-protein interaction network constructed using Cytoscape. Sizes of dots are proportional to the score. Red dots indicate upregulation, blue dots indicate downregulation and gray edges indicate protein interactions.

\section{Discussion}

At present, factors, including heredity, age and mechanical alterations, are involved in the complex pathogenesis of OA (24). The pathogenesis remains unclear, and thus, the treatment of OA primarily relieves symptoms and no specific treatment has been identified. Microarray and sequencing technology that provide expression levels of thousands of genes in humans, have been widely used to predict the potential therapeutic targets for diseases. A thorough understanding of the molecular mechanisms of OA may provide insight for diagnosis and treatment. In the present study, 174 DEGs were identified between synovial membrane with and without inflammation in OA, among which, 145 genes were upregulated and 29 were downregulated. Synovial inflammation has been identified as one of the pathological manifestations of the development and progression of OA (25). These DEGs were involved in the regulation of synovial inflammation. In the present study, an enrichment analysis was conducted to further understand the regulatory roles of DEGs in OA.

The top $10 \mathrm{CC}$ terms of the DEGs demonstrated that they are primarily located in the 'extracellular region', 'plasma membrane', 'vesicles membrane'. The roles of DEGs in MF are to activate 'cytokine activity', promote 'chemokine receptor binding', and regulate 'receptor binding' and 'phospholipid binding'. The top $10 \mathrm{BP}$ terms involved in DEGs are primarily 'inflammatory response', 'immune response' and 'cell-cell signaling'. The KEGG pathways involved in synovial inflammation development of OA by DEGs include 'chemokine signaling pathway', 'complement and coagulation cascades', 'TNF signaling pathway', 'cytokine-cytokine receptor interaction', 'allograft rejection', 'Toll-like receptor signaling pathway' and 'antigen processing and presentation'. Previous studies suggested that immune responses, including complement activation, cytokines and immune cell populations are involved in the occurrence and development of OA (26-28). Additionally, previous studies suggested that cytokines are involved in the pathological process of OA, and cause inflammatory reactions and pain $(29,30)$. A previous study demonstrated that the occurrence of coagulation cascades in the synovium was observed in joint diseases, including OA, and the associated molecules were more highly expressed in inflammatory arthritis (31).

PPI networks containing the DEGs were additionally constructed, and the top 10 hub genes were IL6, IL8, MMP9, CSF1R, FOS, IGF1, TYROBP, MMP3, CD14 and CD163. IL6 and IL8 primarily regulate immune responses and inflammatory responses (32). Previous studies demonstrated that IL6 and IL8 expression levels were increased in OA synovial tissues $(33,34)$. The role of MMP9 is primarily to 
Table I. Enriched pathways of modules 1-3.

A, Module 1

\begin{tabular}{lcl}
\hline Pathway & FDR & \multicolumn{1}{c}{ Genes } \\
\hline Cytokine-cytokine receptor interaction & $2.80 \times 10^{-9}$ & CCL20, CCL8, CSF1R, CXCL1, CXCL16, CXCL2, \\
& & CXCL5, CXCL6, PPBP \\
Rheumatoid arthritis & $2.80 \times 10^{-9}$ & CCL20, CXCL1, CXCL5, CXCL6, FOS, MMP1, MMP3 \\
Chemokine signaling pathway & $3.57 \times 10^{-9}$ & CCL20, CCL8, CXCL1, CXCL16, CXCL2, CXCL5, \\
& & CXCL6, PPBP \\
TNF signaling pathway & $3.57 \times 10^{-9}$ & CCL20, CXCL1, CXCL2, CXCL5, FOS, MMP3, MMP9 \\
Pertussis & $1.35 \times 10^{-6}$ & C1QB, CD14, CXCL5, CXCL6, FOS \\
Transcriptional misregulation in cancer & $8.55 \times 10^{-5}$ & CD14, CSF1R, IGF1, MMP3, MMP9 \\
Salmonella infection & 0.000131 & CD14, CXCL1, CXCL2, FOS \\
Legionellosis & 0.00145 & CD14, CXCL1, CXCL2 \\
Pathways in cancer & 0.00145 & CSF1R, FOS, IGF1, MMP1, MMP9 \\
Complement and coagulation cascades & 0.00248 & BDKRB1, C1QB, SERPINE1 \\
Chagas disease (American trypanosomiasis) & 0.00628 & C1QB, FOS, SERPINE1 \\
Toll-like receptor signaling pathway & 0.00669 & CD14, FOS, SPP1 \\
Osteoclast differentiation & 0.011 & CSF1R, FOS, TYROBP \\
Bladder cancer & 0.0206 & MMP1, MMP9 \\
NOD-like receptor signaling pathway & 0.0439 & CXCL1, CXCL2 \\
\hline
\end{tabular}

B, Module 2

\begin{tabular}{lll}
\hline Pathway & FDR & \\
\hline Cytokine-cytokine receptor interaction & 0.0431 & IL6, IL8, KIT \\
NOD-like receptor signaling pathway & 0.0431 & IL6, IL8 \\
Hematopoietic cell lineage & 0.0431 & IL6, KIT \\
Epithelial cell signaling in Helicobacter pylori infection & 0.0431 & HBEGF, IL8 \\
Salmonella infection & 0.0431 & IL6, IL8 \\
Pertussis & 0.0431 & IL6, IL8 \\
Legionellosis & 0.0431 & IL6, IL8 \\
Malaria & 0.0431 & IL6, IL8 \\
Pathways in cancer & 0.0431 & IL6, IL8, KIT \\
Rheumatoid arthritis & 0.0431 & IL6, IL8 \\
Toll-like receptor signaling pathway & 0.0494 & IL6, IL8 \\
Chagas disease (American trypanosomiasis) & 0.0494 & IL6, IL8 \\
Amoebiasis & 0.0494 & IL6, IL8 \\
\hline
\end{tabular}

C, Module 3

\begin{tabular}{lll}
\hline Pathway & FDR & \\
\hline Intestinal immune network for IgA production & 0.00149 & HLA-DPA1, HLA-DRA \\
Type I diabetes mellitus & 0.00149 & HLA-DPA1, HLA-DRA \\
Staphylococcus aureus infection & 0.00149 & HLA-DPA1, HLA-DRA \\
Asthma & 0.00149 & HLA-DPA1, HLA-DRA \\
Autoimmune thyroid disease & 0.00149 & HLA-DPA1, HLA-DRA \\
Allograft rejection & 0.00149 & HLA-DPA1, HLA-DRA \\
Graft-versus-host disease & 0.00149 & HLA-DPA1, HLA-DRA \\
Viral myocarditis & 0.00152 & HLA-DPA1, HLA-DRA \\
Inflammatory bowel disease & 0.00172 & HLA-DPA1, HLA-DRA \\
Antigen processing and presentation & 0.00175 & HLA-DPA1, HLA-DRA \\
Leishmaniasis & 0.00175 & HLA-DPA1, HLA-DRA
\end{tabular}


Table I. Continued.

C, Module 3

\begin{tabular}{lcc}
\hline Pathway & FDR & Genes \\
\hline Rheumatoid arthritis & 0.00244 & HLA-DPA1, HLA-DRA \\
Systemic lupus erythematosus & 0.00275 & HLA-DPA1, HLA-DRA \\
Toxoplasmosis & 0.00363 & HLA-DPA1, HLA-DRA \\
Cell adhesion molecules & 0.00499 & HLA-DPA1, HLA-DRA \\
Phagosome & 0.0051 & HLA-DPA1, HLA-DRA \\
Tuberculosis & 0.00645 & HLA-DPA1, HLA-DRA \\
Influenza A & 0.00645 & HLA-DPA1, HLA-DRA \\
Herpes simplex infection & 0.00645 & HLA-DPA1, HLA-DRA \\
Epstein-Barr virus infection & 0.00722 & HLA-DPA1, HLA-DRA \\
HTLV-I infection & 0.012 & HLA-DPA1, HLA-DRA \\
\hline
\end{tabular}

FDR, false discovery rate; TNF, tumor necrosis factor; NOD, nucleotide-binding oligomerization domain; IgA, immunoglobulin A; HTLV-I, human T-lymphotrophic like virus I; CCL, C-C motif chemokine ligand; CSF1R, colony stimulating factor 1 receptor; CXCL, C-X-C motif chemokine ligand; PPBP, pro-platelet basic protein; FOS, FOS proto-oncogene, AP-1 transcription factor subunit; MMP, matrix metallopeptidase; C1QB, complement C1q B chain; CD14, CD14 molecule; IGF1, insulin like growth factor 1; BDKRB1, bradykinin receptor 1; SERPINE1, serpin family E member 1; SPP1, secrete phosphoprotein 1; TYROBP, TYRO protein tyrosine kinase binding protein; IL, interleukin; KIT, KIT proto-oncogene receptor tyrosine kinase; HBEGF, heparin binding EGF like growth factor; HLA-DPA1, major histocompatibility complex, class II, DP $\alpha$ 1; HLA-DRA, major histocompatibility complex, class II, DR $\alpha$.

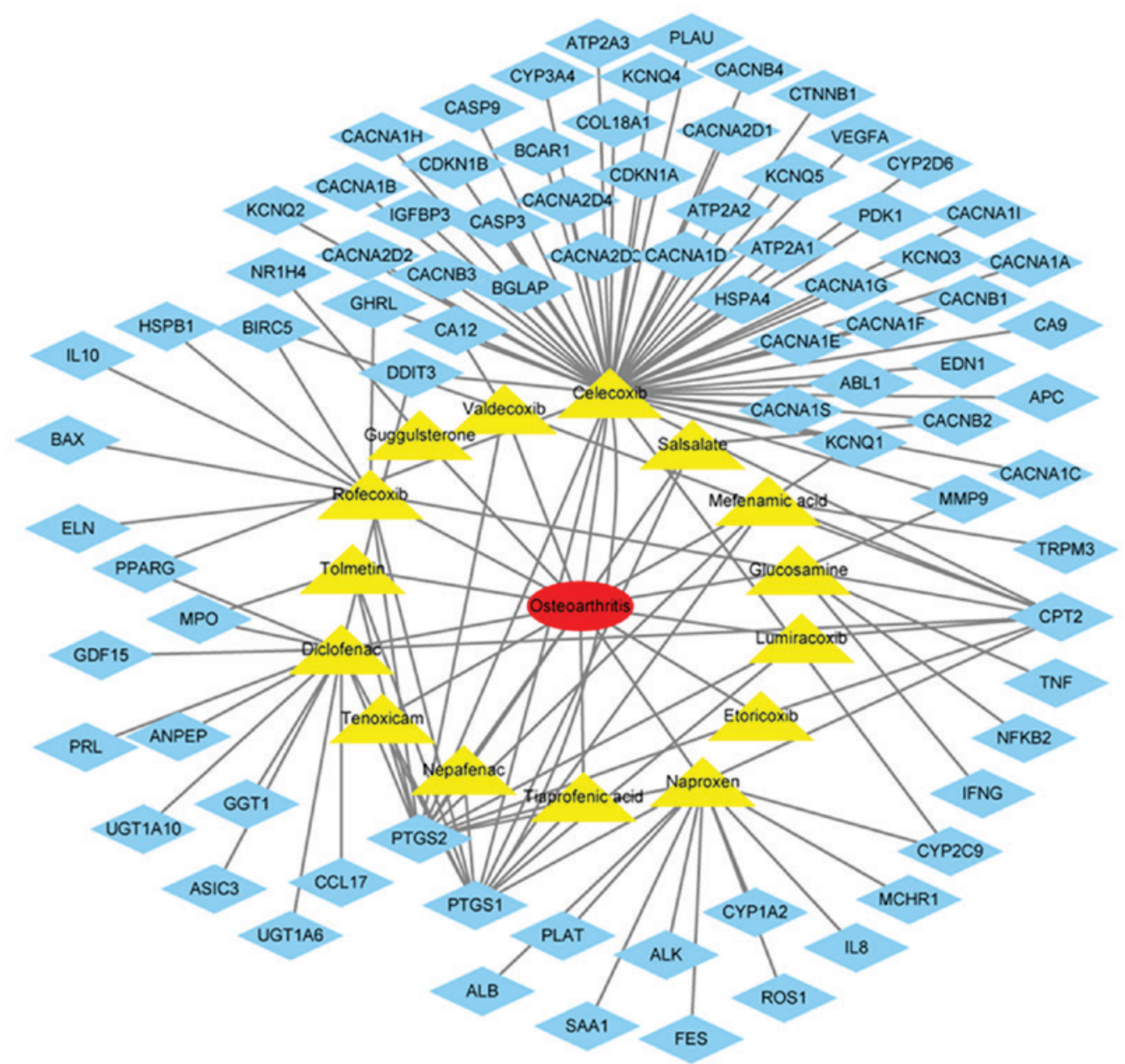

Figure 6. Commonly used drugs for the treatment of osteoarthritis and their target genes. The yellow triangles indicate drugs and the blue diamonds indicate the target genes. 
degrade and remodel the extracellular matrix, and the role of MMP3 is to degrade extracellular matrix proteins (35). The results of the present study were consistent with the results of previous studies, which demonstrated high expression of MMP9 and MMP3 in OA synovial tissues (36,37). CSF1R encodes a receptor protein for colony stimulating factor 1 , which mediates the majority of the biological effects of this cytokine (38). A previous study revealed macrophage-CSF and macrophage-CSF and granulocyte-CSF are involved in the inflammatory response in a rheumatoid arthritis mouse model (39). FOS is a nuclear protein transcription factor that regulates the growth, division, differentiation, proliferation and apoptosis of cells (40). Compared with normal human synovial tissues, immunohistochemistry demonstrated strong staining of c-FOS in OA synovial tissues (41). IGF1 encodes an active protein peptide substance that promotes cell growth and is essential for sugar, lipid, protein metabolism and inorganic salt metabolism (42). A previous study demonstrated that the IGF1 content in OA synovial fluid was twice that in normal fluid and that all forms of IGF1 were highly concentrated in the cartilage of OA, which may affect the progression of the disease by regulating the anabolic metabolism of cartilage macromolecules (43). TYROBP is involved in immune and inflammatory reactions (44). A previous study demonstrated that TYROBP was more highly expressed in OA synovial tissues compared with healthy synovial tissues (45). A previous clinical study demonstrated that the expression levels of CD14 and CD163 in the serum and joint fluid were increased in patients with OA, and were directly proportional to the activation of macrophages, joint space, osteophytes and the severity of pain (46). CD14, as a co-receptor, was involved in the signaling pathway of lipopolysaccharide binding and may trigger inflammatory activation of synovial cells by stimulating toll-like receptors (47). CD163 is a marker for cells from the monocyte/macrophage lineage and is also hemoglobin scavenger receptor, with pro- and anti-inflammatory effects (48). Comparing the results of the present study with the text-mining results, IL8 and MMP9 were identified as useful therapeutic targets for OA.

Module analysis demonstrated that the development of OA synovitis is associated with 'cytokine-cytokine receptor interactions', 'NOD-like receptor signaling pathway', 'Toll-like receptor signaling pathway'. Previous studies have demonstrated that the occurrence and development of OA were associated with a number of cytokines, leading to synovial inflammation, cartilage damage and osteophyte production $(30,49)$. The present study demonstrated that OA synovial inflammation is associated with 'NOD-like receptor signaling pathway', 'Toll-like receptor signaling pathway' and 'TNF signaling pathway', and OA progression is additionally associated with 'osteoclast differentiation'.

In the present study, bioinformatics analysis was performed to investigate synovial inflammation. The DEGs identification and in-depth analysis may help to identify useful targets and pathways in OA pathogenesis. These results may provide insight for the study of OA therapeutic targets. A limitation of the present study was that the screened genes and pathways were not experimentally validated, which may become a focus of future studies.

\section{Acknowledgements}

Not applicable.

\section{Funding}

The present study was funded by the National Natural Science Foundation of China (grant no. 81573801), the Natural Science Foundation of Fujian Province (grant no. 2017J06018) and the 2017 Science and Technology project of Traditional Chinese Medicine of Fujian Province (grant no. 2017FJZYJC204).

\section{Availability of data and materials}

The datasets used and/or analyzed during the current study are available from the corresponding author on reasonable request.

\section{Authors' contributions}

XL and JL conceived and designed the study. GW, ZZ and YH collected the expression data and screened for the differentially expressed genes. JC, CF and JY analyzed and interpreted the data. JL wrote the manuscript. GW and XL reviewed and edited the manuscript. All authors read and approved the final manuscript.

\section{Ethics approval and consent to participate}

Not applicable.

\section{Patient consent for publication}

Not applicable.

\section{Competing interests}

The authors declare that they have no competing interests.

\section{References}

1. McDougall C, Hurd $\mathrm{K}$ and Barnabe C: Systematic review of rheumatic disease epidemiology in the indigenous populations of Canada, the United States, Australia, and New Zealand. Semin Arthritis Rheum 46: 675-686, 2016.

2. Bijlsma JW, Berenbaum F and Lafeber FP: Osteoarthritis: An updata with relevance for clinical practice. Lancet 377 : 2115-2126, 2011.

3. Sulzbacher I: Osteoarthritis: Histology and pathogenesis. Wien Med Wochenschr 163: 212-219, 2013.

4. Hashimoto S, Ochs RL, Komiya S and Lotz M: Linkage of chondrocyte apoptosis and cartilage degradation in human osteoarthritis. Arthritis Rheum 41: 1632-1638, 1998.

5. Schroeppel JP, Crist JD, Anderson HC and Wang J: Molecular regulation of articular chondrocyte function and its significance in osteoarthritis. Histol Histopathol 26: 377-394, 2011.

6. Kleine SA and Budsberg SC: Synovial membrane receptors as therapeutic targets: A review of receptor localization, structure, and function. J Orthop Res, 2017.

7. Wang X, Hunter DJ, Jin X and Ding C: The importance of synovial inflammation in osteoarthritis: Current evidence from imageing assessments and clinical trial. Osteoarthritis Cartilage 26: 165-167, 2018.

8. Bhattaram $P$ and Chandrasekharan $\mathrm{U}$ : The joint synovium: A critical determinant of articular cartilage fate in inflammatory joint diseases. Semin Cell Dev Biol 62: 86-93, 2017. 
9. Scanzello CR and Goldring SR: The role of synovitis in osteoarthritis pathogenesis. Bone 51: 249-257, 2012.

10. Goldenberg DL and Cohen AS: Synovial membrane histopathology in the differential diagnosis of rheumatoid arthritis, gout, pseudogout, systemic lupus erythematosus, infectious arthritis and degenerative joint disease. Medicine (Baltimore) 57 239-252, 1978 .

11. Sellam J and Berenbaum F: The role of synovitis in pathophysiology and clinical symptoms of osteoarthritis. Nat Rev Rheumatol 6: 625-635, 2010 .

12. Myers SL, Brandt KD, Ehlich JW, Braunstein EM, Shelbourne KD, Heck DA and Kalasinski LA: Synovial inflammation in patients with early osteoarthritis of the knee. J Rheumatol 17: 1662-1669, 1990.

13. Ayral X, Pickering EH, Woodworth TG, Mackillop N and Dougados M: Synovitis: A potential predictive factor of structural progression of medial tibiofemoral knee osteoarthritis-results of a 1 year longitudinal arthroscopic study in 422 patients. Osteoarthritis Cartilage 13: 361-367, 2005.

14. Fernandez-Madrid F, Karvonen RL, Teitge RA, Miller PR, An T and Negendank WG: Synovial thickening detected by MR imaging in osteoarthritis of the knee confirmed by biopsy as synovitis. Magn Reson Imaging 13: 177-183, 1995.

15. Felson DT, Niu J, Neoqi T, Goggins J, Nevitt MC, Roemer F, Torner J, Lewis CE and Guermazi A; MOST Investigators Group. Synovitis and the risk of knee osteoarthritis: The MOST Study. Osteoarthritis Cartilage 24: 458-464, 2016.

16. Chen D, Shen J, Zhao W, Wang T, Han L, Hamilton JL and Lm HJ: Osteoarthritis: Toward a comprehensive understanding of pathological mechanism. Bone Res 5: 16044, 2017.

17. Li M, Zhi L, Zhang Z, BIan W and Qiu Y: Identification of potential target genes associated with the pathogenesis of osteoarthritis using microarray based analysis. Mol Med Rep 16 2799-2806, 2017

18. Kong R, Gao J, Si Y and Zhao D: Combination of circulating miR-19b-3p, miR-122-5p and miR-486-5p expressions correlates with risk and disease severity of knee osteoarthritis. Am J Transl Res 9: 2852-2864, 2017.

19. Lambert C, Dubuc JE, Montell E, Verges J, Munaut C, Noel A and Henrotin Y: Gene expression pattern of cell from inflamed and normal areas of osteoarthritis synovial membrane. Arthritis Rheumatol 66: 960-968, 2014

20. Huang DW, Sherman BT and Lempicki RA: Systematic and integrative analysis of large gene lists using DAVID bioinformatics resources. Nat Protoc 4: 44-57, 2009.

21. Szklarczyk D, Morris JH, Cook H, Kuhn M, Wyder S, Simonovic M, Santos A, Doncheva NT, Roth A, Bork P, et al: The STRING database in2017: Quality-controlled protein-protein association networks, made broadly accessible. Nucleic Acids Res 45 (Database Issue): D362-D368, 2017.

22. Bader GD and Hogue CW: An automated method for finding molecular complexes in large protein interaction networks. BMC Bioinformatics 4: 2, 2003.

23. Li YH, Yu CY, Li XX, Zhang P, Tang J, Yang Q, Fu T, Zhang X, Cui X, Tu G, et al: Therapeutic target database update 2018 : Enriched resource for facilitating bench-to-clinic research of targeted therapeutics. Nucleic Acids Res 46: D1121-D1127, 2018.

24. Johnson VL and Hunter DJ: The epidemiology of osteoarthritis. Best Pract Res Clin Rheumatol 28: 5-15, 2014.

25. Benito MJ, Veale DJ, FitzGerald O, van den Berg WB and Bresnihan B: Synovial tissue inflammation in early and late osteoarthritis. Ann Rheum Dis 64: 1263-1267, 2005.

26. Lopes EBP, Filiberti A, Husain SA and Humphrey MB: Immune contributions to osteoarthritis. Curr Osteoporos Rep 15: 593-600, 2017.

27. Kalaitzoglou E, Griffin TM and Humphrey MB: Innate immune responses and osteoarthritis. Curr Rheumatol Rep 19: 45, 2017.

28. Silawal S, Triebel J, Bertsch $\mathrm{T}$ and Schulze-Tanzil G: Osteoarthritis and the complement cascade. Clin Med Insights Arthritis Musculoskelet Disord 11: 1179544117751430, 2018.

29. Syx D, Tran PB, Miller RE and Malfait AM: Peripheral mechanisms contributing to osteoarthritis pain. Curr Rheumatol Rep 20: 9, 2018.
30. Nguyen LT, Sharma AR, Chakraborty C, Saibaba B, Ahn ME and Lee SS: Review of prospects of biological fluid biomarkers in osteoarthritis. Int J Mol Sci 18: pii: E601, 2017.

31. So AK, Varisco PA, Kemkes-Matthes B, Herkenne-Morard C, Chobaz-Peclat V, Gerster JC and Busso N: Arthritis is linked to local and systemic activation of coagulation and fibrinolysis pathways. J Thromb Haemost 1: 2510-2515, 2003.

32. Brocker C, Thompson D, Matsumoto A, Nebert DW and Vasiliou V: Evolutionary divergence and functions of the human interlrukin (IL) gene family. Hum Genomics 5: 30-55, 2010.

33. Yang F, Zhou S, Wang C, Huang Y, Li H, Wang Y, Zhu Z, Tang J and Yan M: Epigenetic modifications of interleukin- 6 in synovial fibroblasts from osteoarthritis patients. Sci Rep 7: 43592, 2017.

34. Nair A, Gan J, Bush-Joseph C, Verma N, Tetreault MW, Saha K, Margulis A, Fogg L and Scanzello CR: Synovial chemokine expression and relationship with knee symptoms in patients with meniscal tears. Osteoarthritis Cartilage 23: 1158-1164, 2015.

35. Rohani MG and Parks WC: Matrix remodeling by MMps during wound repair. Matrix Biol 44-46: 113-121, 2015.

36. Kanyama M, Kuboki T, Kojima S, Fujisawa T, Hattori T, Takigawa $\mathrm{M}$ and Yamashita A: Matrix metalloproteinases and tissue inhibitors of metalloproteinases in synovial fluids of patients with temporomandibular joint osteoarthritis. J Orofac Pain 14: 20-30, 2000.

37. Yang CC, Lin CY, Wang HS and Lyu SR: Matrix metalloproteases and tissue inhibitors to knee osteoarthritis progression. PLoS One 8: e79662, 2013.

38. Meyers MJ, Pelc M, Kamtekar S, Day J, Poda GI, Hall MK, Michener ML, Reitz BA, Mathis KJ, Pierce BS, et al: Structure-based drug design enables conversion of a DFG-in bingding CSF-1R kinase inhibitor to a DFG-out binding mode. Bioorg Med Chem Lett 20: 1543-1547, 2010.

39. Campbell IL, Rich MJ, Bioschof RJ and Hamilton JA: The colony-stimulating factors and collagen-induced arthritis: Exacerbation of disease by M-CSF and G-CSF and requirement for endogenous M-CSF. J Leukoc Biol 68: 144-150, 2000.

40. Chung L: A brief introduction to the transduction of neural activity into fos signal. Dev Reprod 19: 61-67, 2015.

41. Kinne RW, Boehm S, Iftner T, Aigner T, Vornehm S, Weseloh G, Bravo R, Emmrich F and Kroczek RA: Synovial fibroblast-like cells strongly express jun-B and C-fos proto-oncogenes in rheumatoid- and osteoarthritis. Scand J Rheumatol Suppl 101: 121-125, 1995.

42. Annunziata M, Granata R and Ghiqo E: The IGF system. Acta Diabetol 48: 1-9, 2011.

43. Schneiderman R, Rosenberg N, Hiss J, Lee P, Liu F, Hintz RL and Maroudas A: Concentration and size distribution of insulin-like growth factor-1 in human normal and osteoarthritis synovial fluid and cartilage. Arch Biochem Biophys 324: 173-188, 1995.

44. Tomasello E and Vivier E: KARAP/DAP12/TYROBP Three names and a multiplicity of biological functions. Eur J Immunol 35: 1670-1677, 2005.

45. Crotti YN, Dharmapatni AA, Alias E, Zannettino AC, Smith MD and Haynes DR: The immunoreceptor tyrosine-based activation motif (IYAM)-related factors are increased in synovial tissue and vasculature of rheumatoid arthritic joints. Arthritis Res Ther 14: R245, 2012.

46. Daghestani HN, Pieper CF and Kraus VB: Solube macrophage biomarkers indicate inflammatory phenotypes in patients with knee osteoarthritis. Arthritis Rheumatol 67: 956-965, 2015.

47. Nair A, Kanda V, Bush-Joseph C, Verma N, Chubinskaya S, Mikecz K, Glant TT, Malfair AM, Crow MK, Spear GT, et al: Synovial fluid from patients with early osteoarthritis modulates fibroblast-like synoviocyte responses to toll-like recepyor 4 and toll-like receptor 2 ligands via soluble CD14. Arthritis Rheum 64: 2268-2277, 2012.

48. Fabriek BO, Dijkstra CD and van den Berg TK: The macrophage scavenger receptor CD163. Immunobiology 210: 153-160, 2005.

49. Kim JR, Yoo JJ and Kim HA: Therapeutics in osteoarthritis based on an understanding of its molecular pathogenesis. Int J Mol Sci 19: E674, 2018

This work is licensed under a Creative Commons Attribution-NonCommercial-NoDerivatives 4.0 International (CC BY-NC-ND 4.0) License. 